\title{
Accurate and Linear Time Pose Estimation from Points and Lines
}

\author{
Alexander Vakhitov ${ }^{1}$ Jan Funke ${ }^{2}$ Francesc Moreno-Noguer ${ }^{2}$ \\ ${ }^{1}$ Department of Mathematics and Mechanics, St. Petersburg University, Russia \\ ${ }^{2}$ Institut de Robòtica i Informàtica Industrial, UPC-CSIC, Barcelona, Spain \\ a.vakhitov@spbu.ru, \{jfunke,fmoreno\}@iri.upc.edu
}

\begin{abstract}
The Perspective- $n$-Point (PnP) problem seeks to estimate the pose of a calibrated camera from $n 3 \mathrm{D}$-to-2D point correspondences. There are situations, though, where PnP solutions are prone to fail because feature point correspondences cannot be reliably estimated (e.g. scenes with repetitive patterns or with low texture). In such scenarios, one can still exploit alternative geometric entities, such as lines, yielding the so-called Perspective- $n$-Line (PnL) algorithms. Unfortunately, existing PnL solutions are not as accurate and efficient as their point-based counterparts. In this paper we propose a novel approach to introduce 3D-to-2D line correspondences into a PnP formulation, allowing to simultaneously process points and lines. For this purpose we introduce an algebraic line error that can be formulated as linear constraints on the line endpoints, even when these are not directly observable. These constraints can then be naturally integrated within the linear formulations of two state-of-the-art point-based algorithms, the $\mathrm{OPnP}$ [45] and the EPnP [24], allowing them to indistinctly handle points, lines, or a combination of them. Exhaustive experiments show that the proposed formulation brings remarkable boost in performance compared to only point or only line based solutions, with a negligible computational overhead compared to the original OPnP and EPnP.
\end{abstract}

\section{Introduction}

The objective of the Perspective- $n$-Point problem $(\mathrm{PnP})$ is to estimate the pose of a calibrated camera from $n$ known 3D-to-2D point correspondences [34]. Early approaches were focused on solving the problem for the minimal cases with $n=\{3,4,5\}[7,11,12,15,19,42]$. The proliferation of feature point detectors $[16$, $36]$ and descriptors $[3,26,29,37,40]$ able to consistently retrieve many feature points per image, brought a series of new $\mathrm{PnP}$ algorithms that could efficiently handle arbitrarily large sets of points $[9,13,18,24,25,27,30,38,45]$. Amongst

This work is partly funded by the Russian MES grant RFMEFI61516X0003; by the Spanish MINECO project RobInstruct TIN2014-58178-R and by the ERA-Net Chistera project I-DRESS PCIN-2015-147. 

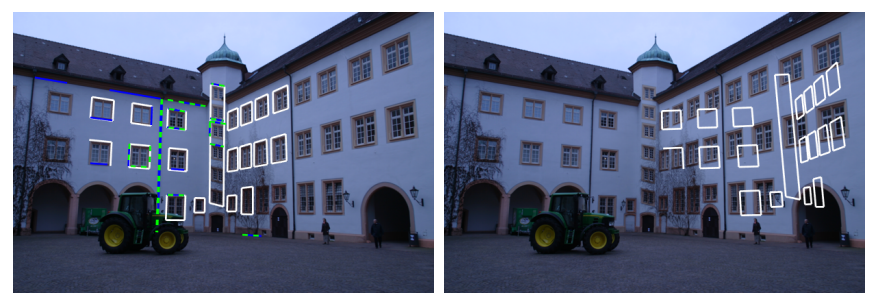

Fig. 1. Pose estimation results of OPnPL (left) and OPnP (right) in a scenario with a lack of reliable feature points. Blue points and solid line segments are detected in the image, and green dashed line segments are the model reference lines reprojected using the estimated pose. White lines are manually chosen on the 3D model to sketch its structure and projected onto the image to deem the quality of the estimated pose. Note also the shift along the line direction between the detected and the model lines. This issue needs to be handled in practice, where the reference lines in the model may only be partially detected on the image (due to partial occlusions). Images from [41].

them, it is worth highlighting the EPnP [24], the first of these 'efficient' PnP solutions, and the OPnP [45], one of the most recent and accurate alternatives.

However, there are situations were $\mathrm{PnP}$ algorithms are likely to perform poorly because the presence of repetitive patterns or a lack of texture makes it difficult to reliably estimate and match feature points. This occurs, for instance, in man-made structures such as that shown in Fig. 1. In these cases, though, one can still rely on other geometric primitives like straight lines, and compute camera pose using the so-called Perspective-n-Line (PnL) algorithms. Unfortunately, existing solutions are not yet as accurate as the point based approaches. The main problem to tackle in the PnL is that even when one may know 3Dto-2D line correspondences, there still can exist a shift of the lines along their direction. Additionally, the line may only be partially observed due to occlusions or misdetections (see again Fig. 1).

In this paper, we propose a formulation of the PnL problem which is robust to partial and shifted line observations. At the core of our approach lies a parameterization of the algebraic line segment reprojection error, that is linear on the segment endpoints. This parameterization, in turn, can be naturally integrated within the formulation of the original EPnP and OPnP algorithms, hence, allowing these PnP methods to leverage information about line correspondences with an almost negligible extra computation. We denote these joint line and point formulations as EPnPL and OPnPL, and through an exhaustive experimentation, show that they consistently improve the point only, and line only formulations.

\section{Related Work}

Most camera pose estimation algorithms are based on either point or line correspondences. Only a few works exploit lines' and points' projections simultaneously. We next review the main related work in each of these categories. 
Pose from Points $(\mathbf{P} n \mathbf{P})$. The most standard approach for pose estimation considers 3D-to-2D point correspondences. Early PnP approaches addressed the minimal cases, with solutions for the P3P $[6,12,14,21], \mathrm{P} 4 \mathrm{P}[4,11]$ and $n=$ $\{4,5\}[42]$. These solutions, however, were by construction unable to handle larger amounts of points, or if they could, they were computationally demanding. Arbitrary number of points can be handled by the Direct Linear Transformation (DLT) [1], which however, estimates the full projection matrix without exploiting the fact that the internal parameters of the camera are known. This knowledge is shown to improve the pose estimation in more recent $\mathrm{PnP}$ approaches focused on building efficient solutions for the overconstrained case $[2,10,18,20,24,32,45]$. Amongst these, the EPnP [24] was the first $O(n)$ solution. Its main idea was to represent the $3 \mathrm{D}$ point coordinates as a linear combination of four control points, which became the only unknowns of the problem, independently of the total number of 3D coordinates. These control points were then retrieved using simple linearization techniques. This linearization has been subsequently substituted by polynomial solvers in the Robust PnP (RPnP) [25], the Direct Least Squares DLS [18], and the Optimal PnP (OPnP) [45], the most accurate of all PnP solutions. OPnP, draws inspiration on the DLS, but by-passes a degeneracy singularity of the DLS on the rotation by using a quaternion parameterization that allows to directly estimate the pose using a Gröbner basis solver.

Pose from Lines $(\mathbf{P} n \mathbf{L})$. The number of PnL algorithms is considerably smaller than the point-based ones. Back in the 90's, closed-form approaches for the minimal case with 3 line correspondences were proposed [5,7], together with theoretical studies about the multiple solutions this problem could have [31]. The DLT was shown to be applicable to line representations in [17], although again, with poorer results than algorithms that explicitly exploited the knowledge of the internal parameters of the camera, like [2], also applied to lines. [28] estimates the camera rotation matrix by solving a polynomial system of equations using an eigendecomposition of a so-called multiplication matrix. This method has been recently extended to full pose estimation (rotation+translation) in [33], by combining Pluecker 3D line parameterization with a DLT-like estimation algorithm. [44] combines the former P3L algorithms to compute pose by optimizing a cost function built from line triplets. Finally, [23] shows promising results by formulating the problem in terms of a system of symmetric polynomials.

Pose from Points and Lines $(\mathbf{P} n \mathbf{P L})$. There is a very limited number of approaches that can simultaneously process point and line correspondences. The first of these approaches is the aforementioned DLT, initially used for point based pose estimation [1], and later extended to lines [17]. Both formulations can be integrated into the same framework. [8] also claims a methodology that can potentially handle points and lines. Unfortunately, this is not theoretically shown neither demonstrated in practice. Finally, there are a few works that tackle the camera pose estimation from minimal number of points and lines. [35] proposes solutions for the P3L, P2L1P, P1L2P and P3L. And most recently, [22] solves for pose and focal length from points, directions and points with directions. 


\section{Our Approach to Pose from Points and Lines}

Our approach to pose estimation holds on a new formulation of the straight line projection error, which allows incorporating information about the matched line segments into the $\mathrm{PnP}$ method, and in particular, into the $\mathrm{EPnP}$ and $\mathrm{OPnP}$. This will result in two new algorithms for pose estimation from point and line correspondences, denoted as EPnPL and OPnPL.

\subsection{Problem Formulation}

We are given $n$ correspondences between $3 \mathrm{D}$ reference lines and $2 \mathrm{D}$ segment projections. 3D lines are represented by $3 \mathrm{D}$ endpoints $\left\{\mathbf{P}^{i}, \mathbf{Q}^{i}\right\}$ and $2 \mathrm{D}$ detected segments by $2 \mathrm{D}$ endpoints $\left\{\mathbf{p}_{\mathrm{d}}^{i}, \mathbf{q}_{\mathrm{d}}^{i}\right\}$, for $i=1, \ldots, n$. The camera is assumed to be calibrated, being $\mathrm{K}$ the matrix of internal parameters. Our goal is to estimate the rotation $R$ and translation $\mathbf{t}$ that align the camera and the world coordinate frames. It is worth pointing out that $2 \mathrm{D}$ line segment endpoints $\mathbf{p}_{\mathrm{d}}^{i}, \mathbf{q}_{\mathrm{d}}^{i}$ do not necessarily correspond to the projections $\mathbf{p}^{i}, \mathbf{q}^{i}$ of the $3 \mathrm{D}$ line endpoints $\mathbf{P}^{i}, \mathbf{Q}^{i}$. They are, instead, projections of some points $\mathbf{P}_{\mathrm{d}}^{i}, \mathbf{Q}_{\mathrm{d}}^{i}$ lying on the same $3 \mathrm{D}$ line as $\mathbf{P}^{i}, \mathbf{Q}^{i}$ (see Fig. 2-left). This reflects the fact that in practice 3D reference lines may not be fully detected in the image or they can be partially occluded, precluding the use of point-based $\mathrm{PnP}$ algorithms.

\subsection{General Definition of Line Segment Projection Error}

Let the vector $\boldsymbol{\theta}$ denote the pose parameters ( $\mathrm{R}$ and $\mathbf{t}$ ) of the calibrated camera. In the $\mathrm{PnP}$ formulation, we minimize the reprojection error of a projection function $\tilde{\mathbf{x}}=\pi(\boldsymbol{\theta}, \mathbf{X})$, where $\tilde{\mathbf{x}} \in \mathbb{R}^{3}$ are the homogeneous coordinates of the $3 \mathrm{D}$ point $\mathbf{X}$ projected on the camera. Since we assume the calibration matrix $\mathrm{K}$ is known, we can pre-multiply the homogeneous image plane coordinates of the detected lines and points by $\mathrm{K}^{-1}$ prior to solving the PnPL problem. In the rest of this document, we will therefore assume that the homogeneous coordinates are normalized and that $\mathrm{K}$ is a identity matrix.

In order to extend the point-based formulation to handle line segments, we need to formalize the reprojection error for lines. For that, let $\tilde{\mathbf{p}}_{\mathrm{d}}^{i}, \tilde{\mathbf{q}}_{\mathrm{d}}^{i} \in \mathbb{R}^{3}$ be the homogeneous coordinates of the detected $2 \mathrm{D}$ endpoints $\mathbf{p}_{\mathrm{d}}^{i}, \mathbf{q}_{\mathrm{d}}^{i}$ for the $i$-th line segment. We represent this projected segment by its normalized line coefficients:

$$
\hat{\mathbf{l}}^{i}=\tilde{\mathbf{p}}_{\mathrm{d}}^{i} \times \tilde{\mathbf{q}}_{\mathrm{d}}^{i}, \quad \mathbf{l}^{i}=\frac{\hat{\mathbf{l}}^{i}}{\left|\hat{\mathbf{l}}^{i}\right|} \in \mathbb{R}^{3} .
$$

We then define the algebraic point-line error $\mathrm{E}_{\mathrm{pl}}$ for a $3 \mathrm{D}$ point $\mathbf{P}^{i}$ to a detected line segment $\mathbf{l}^{i}$ as distance between the line $\mathbf{l}^{i}$ and the $2 \mathrm{D}$ projection of $\mathbf{P}^{i}$ :

$$
\mathrm{E}_{\mathrm{pl}}\left(\boldsymbol{\theta}, \mathbf{P}^{i}, \mathbf{l}^{i}\right)=\left(\mathbf{l}^{i}\right)^{\top} \pi\left(\boldsymbol{\theta}, \mathbf{P}^{i}\right) .
$$



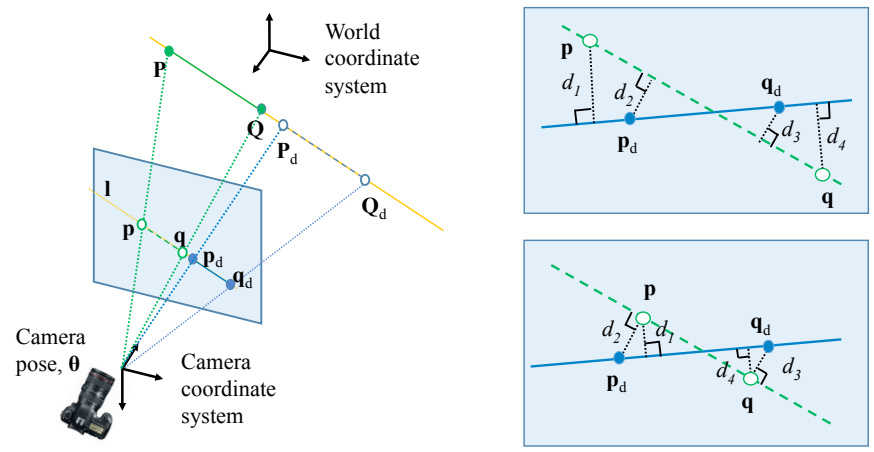

Fig. 2. Left: Notation and problem formulation. Given a set of 3D-to-2D line correspondences, we seek to estimate the pose $\boldsymbol{\theta}$ that aligns the camera and the world coordinate systems. 3D lines are represented by $3 \mathrm{D}$ endpoint pairs $(\mathbf{P}, \mathbf{Q})$. 2D corresponding segments are represented by the detected endpoints $\left(\mathbf{p}_{\mathrm{d}}, \mathbf{q}_{\mathrm{d}}\right)$ in the image plane. Note that the 3D-to-2D line correspondence does not imply a correspondence of the enpdoints, preventing the use of a standard PnP algorithm. Right: Correction of $3 \mathrm{D}$ line segments to put in correspondence the projection of the line endpoints $(\mathbf{P}, \mathbf{Q})$ with the detected segment endpoints $\left(\mathbf{p}_{\mathrm{d}}, \mathbf{q}_{\mathrm{d}}\right)$. Top: before correction. Bottom: after correction. Note how the projections $(\mathbf{p}, \mathbf{q})$ have been shifted along the line.

We further define the algebraic line segment error $\mathrm{E}_{\mathrm{l}}$ as the sum of squares of the two point-line errors for the 3D line segment endpoints:

$$
\mathrm{E}_{\mathbf{l}}\left(\boldsymbol{\theta}, \mathbf{P}^{i}, \mathbf{Q}^{i}, \mathbf{l}^{i}\right)=\mathrm{E}_{\mathrm{pl}}^{2}\left(\boldsymbol{\theta}, \mathbf{P}^{i}, \mathbf{l}^{i}\right)+\mathrm{E}_{\mathrm{pl}}^{2}\left(\boldsymbol{\theta}, \mathbf{Q}^{i}, \mathbf{l}^{i}\right) .
$$

The overall line segment error $\mathrm{E}_{\text {lines }}$ for the whole image is the accumulated algebraic line segment error over all the matched line segments:

$$
\mathrm{E}_{\text {lines }}\left(\boldsymbol{\theta},\left\{\mathbf{P}^{i}\right\},\left\{\mathbf{Q}^{i}\right\},\left\{\mathbf{l}^{i}\right\}\right)=\sum_{i} \mathrm{E}_{\mathbf{l}}\left(\boldsymbol{\theta}, \mathbf{P}^{i}, \mathbf{Q}^{i}, \mathbf{l}^{i}\right) .
$$

Note that this error does not explicitly use the detected line segment endpoints, depending only on the line coefficients $\mathbf{l}^{i}$. However, we seek to approximate with it the distance between the detected endpoints and the line projected from the model onto the image plane. This approximation may incur in gross errors in situations such as the one depicted in Fig.2-top-right. In this scenario, the true projected endpoints $(\mathbf{p}, \mathbf{q})$ are relatively far from the $2 \mathrm{D}$ detected endpoints $\left(\mathbf{p}_{\mathrm{d}}, \mathbf{q}_{\mathrm{d}}\right)$, and the algebraic point-line errors $d_{1}$ and $d_{4}$ are much larger than the gold standard errors $d_{2}, d_{3}$. This leads to preferred minimization of the algebraic error for line matches where the detected and projected endpoints are further away. To handle this problem we considered a two-step approach. We first estimate the initial camera pose with the given 3D model. We then recompute the position of the end-points onto the $3 \mathrm{D}$ model such that they reduce the distances between the projected and detected endpoints. Using this updated 3D model, 
we compute the final estimate of the camera pose. Fig.2-bottom-right shows how the ground truth projected end-points have changed their position after having updated the 3D model. We next describe in more detail this correction process.

\subsection{Putting 3D and 2D Line Endpoints in Correspondence}

Let's consider $d$ be the length of the detected line segment and the notation detailed in Fig. 2-right. After the first iteration of the complete PnPL algorithm (see Sections 3.4 and 3.5) we obtain an initial estimate of the camera pose. We then shift the endpoints of every line segment in the camera coordinate frame so that the length of the projected line segment matches the length of the detected segments, and the sum of distances between corresponding endpoints is minimal.

More specifically, given an estimate for the pose $\mathrm{R}, \mathbf{t}$, we compute $\mathbf{p}, \mathbf{q}$ and the unit line direction vector $\mathbf{v}$ along the projected line $\hat{\mathbf{l}}$. We then shift the position of $\mathbf{p}$ and $\mathbf{q}$ along this line, such that they become as close as possible from $\mathbf{p}_{\mathrm{d}}, \mathbf{q}_{\mathrm{d}}$, and separated by a distance $d$. This can be expressed with the following two equations, function of a shifting parameter $\gamma$ :

$$
\mathbf{p}_{\mathrm{d}}=\mathbf{p}+\gamma \mathbf{v} \quad \mathbf{q}_{\mathrm{d}}=\mathbf{p}+(\gamma+d) \mathbf{v}
$$

which yields that $\gamma=\mathbf{v}^{\top}\left(\frac{1}{2}\left(\mathbf{p}_{\mathrm{d}}+\mathbf{q}_{\mathrm{d}}\right)-\mathbf{p}\right)-\frac{d}{2}$. Given $\gamma$ we can then take the right hand side of (5) as the new projections of $\mathbf{p}, \mathbf{q}$, and backproject the position of the new endpoints $\mathbf{P}, \mathbf{Q}$ in the camera and world coordinate frames.

To backproject a point from the image plane to a $3 \mathrm{D}$ line, we compute the intersection of the line of sight of the point with the 3D line as follows:

$$
\lambda \tilde{\mathbf{x}}=\alpha \mathbf{X}+\beta \mathbf{D},
$$

where $\tilde{\mathbf{x}}$ are the point's projection homogeneous coordinates, $\mathbf{X}$ is the $3 \mathrm{D}$ point belonging to the line and $\mathbf{D}$ is the $3 \mathrm{D}$ line direction. Both $\mathbf{X}$ and $\mathbf{D}$ are expressed w.r.t. the camera coordinate frame. From this equation we see that $\mathbf{s}=[-\lambda, \alpha, \beta]^{\top}$ is orthogonal to the vectors $[\mathbf{X}(j), \mathbf{D}(j),-\tilde{\mathbf{x}}(j)]^{\top}, j=1,2$, where $\mathbf{X}(j)$ corresponds to the $j$-th component of the vector $\mathbf{X}$. We employ the cross product operation to solve for $\mathbf{s}$ and then compute the $3 \mathrm{D}$ point position as $\mathbf{X}+\frac{\beta}{\alpha} \mathbf{D}$. This procedure turns to be very fast.

\subsection{EPnPL}

We next describe a necessary modification to the EPnP algorithm to simultaneously consider $n_{\mathrm{p}}$ point and $n_{\mathrm{l}}$ line correspondences.

In the EPnP [24] the projection of a point on the camera plane is written as

$$
\pi_{\mathrm{EPnP}}(\boldsymbol{\theta}, \mathbf{P})=\mathrm{K} \sum_{j=1}^{4} \alpha_{j} \mathbf{C}_{j}^{c},
$$

where $\alpha_{j}$ are point-specific coefficients computed from the model and $\mathbf{C}_{j}^{c}$ for $j=1, \ldots, 4$ are the unknown control point coordinates in the camera frame. 
Recall that we are considering normalized homogeneous coordinates, and hence we can set the calibration matrix $\mathrm{K}$ to be the identity matrix. We define our vector of unknowns as $\boldsymbol{\mu}=\left[\mathbf{C}_{1}^{\top}, \mathbf{C}_{2}^{\top}, \mathbf{C}_{3}^{\top}, \mathbf{C}_{4}^{\top}\right]^{\top}$ and then obtain, using (2), an expression for the algebraic point-line error in case of EPnP:

$$
\mathrm{E}_{\mathrm{pl}, \operatorname{EPnP}}\left(\boldsymbol{\theta}, \mathbf{P}^{i}, \mathbf{l}^{i}\right)=\sum_{j=1}^{4} \alpha_{j}\left(\mathbf{l}^{i}\right)^{\top} \mathbf{C}_{j}^{c}=\left(\mathbf{m}_{l}^{i}\left(\mathbf{P}^{i}\right)\right)^{\top} \boldsymbol{\mu},
$$

for $\mathbf{m}_{l}^{i}\left(\mathbf{P}^{i}\right)=\left(\left[\alpha_{1}, \alpha_{2}, \alpha_{3}, \alpha_{4}\right] \otimes \mathbf{l}^{i}\right)^{\top}$. The overall error in Eq. 4 then becomes

$$
\mathrm{E}_{\text {lines }}\left(\boldsymbol{\theta},\left\{\mathbf{P}^{i}\right\},\left\{\mathbf{Q}^{i}\right\},\left\{\mathbf{l}^{i}\right\}\right)=\sum_{i}\left(\left(\mathbf{m}_{l}^{i}\left(\mathbf{P}^{i}\right)\right)^{\top} \boldsymbol{\mu}\right)^{2}+\left(\left(\mathbf{m}_{l}^{i}\left(\mathbf{Q}^{i}\right)\right)^{\top} \boldsymbol{\mu}\right)^{2} .
$$

Finally, considering both point and lines correspondences, the function to be minimized by the EPnPL will be

$$
\underset{\boldsymbol{\mu}}{\arg \min }\left\{\left\|\mathrm{M}_{\mathrm{p}} \boldsymbol{\mu}\right\|^{2}+\mathrm{E}_{\text {lines }}\left(\boldsymbol{\theta},\left\{\mathbf{P}^{i}\right\},\left\{\mathbf{Q}^{i}\right\},\left\{\mathbf{l}^{i}\right\}\right)\right\}=\underset{\boldsymbol{\mu}}{\arg \min }\left\{\|\overline{\mathrm{M}} \boldsymbol{\mu}\|^{2}\right\}
$$

where $\overline{\mathrm{M}}=\left[\mathrm{M}_{\mathrm{p}}^{\top}, \mathrm{M}_{1}^{\top}\right]^{\top} \in \mathbb{R}^{2\left(n_{\mathrm{p}}+n_{1}\right) \times 12}, \mathrm{M}_{\mathrm{p}} \in \mathbb{R}^{2 n_{\mathrm{p}} \times 12}$ is the matrix of parameters for the $n_{\mathrm{p}}$ point correspondences, as in [24], and $\mathrm{M}_{\mathrm{l}} \in \mathbb{R}^{2 n_{1} \times 12}$ is the matrix corresponding to the point-line errors of the $n_{1}$ matched line segments. Equation 10 is finally minimized by following the EPnP methodology.

\subsection{OPnPL}

In $\mathrm{OPnP}$ [45], the camera parameters are represented as $\hat{\mathrm{R}}=\frac{1}{\lambda} \mathrm{R}, \hat{\mathbf{t}}=\frac{1}{\lambda} \mathbf{t}$, where $\bar{\lambda}$ is an average point depth in the camera frame. To deal with points and lines we define $\bar{\lambda}$ as the average depth of the points and line segments' endpoints. Similarly, we compute the mean point $\overline{\mathbf{Q}}$, which can be used to write the third component of $\hat{\mathbf{t}}$ :

$$
\hat{t}_{3}=1-\hat{\mathbf{r}}_{3}^{\top} \overline{\mathbf{Q}}
$$

where $\hat{\mathbf{r}}_{3}$ is the third row of $\hat{\mathrm{R}}$. The projection of a $3 \mathrm{D}$ point $\mathbf{X}$ onto the image plane (assuming the calibration matrix $\mathrm{K}$ to be the identity) will be:

$$
\pi_{\mathrm{OPnP}}(\boldsymbol{\theta}, \mathbf{X})=\hat{\mathrm{R}} \mathbf{X}+\hat{\mathbf{t}} .
$$

As we did for the EPnP, we use this projection into Eq. 2 to compute the algebraic point-line error.

Following [45], we can use Eq. 11 for $\hat{t}_{3}$ to compute the algebraic error for all points $\mathrm{E}_{\text {points }}$ as a function of $\hat{\mathrm{R}}, \hat{t}_{1}, \hat{t}_{2}$ :

$$
\mathrm{E}_{\text {points }}(\hat{\mathbf{r}}, \hat{\mathbf{t}})=\left\|\mathrm{G}_{\mathrm{p}} \hat{\mathbf{r}}+\mathrm{H}_{\mathrm{p}} \hat{\mathbf{t}}_{12}+\mathbf{k}_{\mathrm{p}}\right\|^{2},
$$

where $\hat{\mathbf{r}}$ is a vectorized form of $\hat{\mathrm{R}}, \hat{\mathbf{t}}_{12}=\left[\hat{t}_{1} \hat{t}_{2}\right]^{\top}, \mathrm{G}_{\mathrm{p}} \in \mathbb{R}^{2 n_{\mathrm{p}} \times 9}$ and $\mathrm{H}_{\mathrm{p}} \in \mathbb{R}^{2 n_{\mathrm{p}} \times 2}$ are matrices built from the projections and $3 \mathrm{D}$ model coordinates of the $n_{\mathrm{p}}$ points, and $\mathbf{k}_{\mathrm{p}}$ is a $2 n_{\mathrm{p}}$ constant vector. 
The overall line segment error $\mathrm{E}_{\text {lines }}$ as defined in Eq. 4 can be expressed in the same form as in Eq. 13 with $\mathrm{G}_{\mathrm{l}}, \mathrm{H}_{\mathrm{l}}, \mathbf{k}_{\mathrm{l}}$ instead of $\mathrm{G}_{\mathrm{p}}, \mathrm{H}_{\mathrm{p}}, \mathbf{k}_{\mathrm{p}}$, and using algebraic line segment error (3) with (12) as point-line projection function.

In order to compute the pose, we adapt the cost function of [45] to the following one with both points and lines terms:

$$
\mathrm{E}_{\text {tot }}(\boldsymbol{\rho}, \hat{\mathbf{t}})=\mathrm{E}_{\text {points }}(\hat{\mathbf{r}}(\boldsymbol{\rho}), \hat{\mathbf{t}})+\mathrm{E}_{\text {lines }}(\hat{\mathbf{r}}(\boldsymbol{\rho}), \hat{\mathbf{t}}),
$$

where we parameterize $\hat{\mathbf{r}}$ with non-unit quaternions vector $\boldsymbol{\rho}=[a, b, c, d]^{\top}$. We next seek to minimize this function w.r.t. the pose parameters.

Setting the derivative of $E_{\text {tot }}$ w.r.t. $\hat{\mathbf{t}}$ to zero, and denoting $G_{\text {tot }}=\left[G_{p}^{\top}, G_{1}^{\top}\right]^{\top}$, $\mathrm{H}_{\mathrm{tot}}=\left[\mathrm{H}_{\mathrm{p}}^{\top}, \mathrm{H}_{1}^{\top}\right]^{\top}, \mathbf{k}_{\mathrm{tot}}=\left[\mathbf{k}_{\mathrm{p}}^{\top}, \mathbf{k}_{1}^{\top}\right]^{\top}$ we can write $\hat{\mathbf{t}}$ as a function of $\hat{\mathbf{r}}$ :

$$
\mathrm{H}_{\text {tot }}^{\top}\left(\mathrm{G}_{\mathrm{tot}} \hat{\mathbf{r}}+\mathrm{H}_{\mathrm{tot}} \hat{\mathbf{t}}+\mathbf{k}_{\mathrm{tot}}\right)=0 \quad \Longrightarrow \hat{\mathbf{t}}=\mathrm{P} \hat{\mathbf{r}}+\mathbf{u},
$$

for $\mathrm{P}=-\left(\mathrm{H}_{\text {tot }}^{\top} \mathrm{H}_{\text {tot }}\right)^{-1}\left(\mathrm{H}_{\text {tot }}^{\top} \mathrm{G}_{\text {tot }}\right), \mathbf{u}=-\left(\mathrm{H}_{\mathrm{tot}}^{\top} \mathrm{H}_{\text {tot }}\right)^{-1} \mathrm{H}_{\text {tot }}^{\top} \mathbf{k}_{\text {tot }}$.

The derivative of $\mathrm{E}_{\mathrm{tot}}$ w.r.t. the first quaternion parameter $a$ is:

$$
\frac{\partial \hat{\mathbf{r}}}{\partial a} G_{\text {tot }}^{\top}\left(G_{\text {tot }} \hat{\mathbf{r}}+\mathrm{H}_{\text {tot }} \hat{\mathbf{t}}+\mathbf{k}_{\text {tot }}\right)=\frac{\partial \hat{\mathbf{r}}}{\partial a} G_{\text {tot }}^{\top}\left(\left(G_{\text {tot }}+\mathrm{H}_{\text {tot }} \mathrm{P}\right) \hat{\mathbf{r}}+\mathrm{H}_{\text {tot }} \mathbf{u}+\mathbf{k}_{\text {tot }}\right)=0,
$$

where in the second step we have used Eq. 15. Three more equations analogous to (16) constitute a system which the original OPnP uses to design a specific polynomial solver [45]. In our case we can use exactly the same solver, as the equations we get for joint point and line matches, have exactly the same form as when only considering points.

\section{Experiments}

We evaluate the proposed approach in "points and lines" and "only lines" situations, nonplanar and planar configurations, and synthetic and real experiments.

\subsection{Synthetic Experiments}

We will compare our approach against state-of-the-art in two situations: A joint point-and-line case, and a line-only case. In both scenarios we will consider nonplanar and planar configurations, and will report rotation and translation errors for increasing amounts of 2D noise and number of correspondences. We will also study the influence of the amount of shift between the lines in the 3D model, and the observed segments projected on the image.

In the synthetic experiments we assume a virtual $640 \times 480$ pixels calibrated camera with focal length of 500 . We randomly generate $n_{\mathrm{p}}+2 n_{\mathrm{l}} 3 \mathrm{D}$ points in a box with coordinates $[-2,2] \times[-2,2] \times[4,8]$ in the camera frame, where $n_{\mathrm{p}}$ and $n_{1}$ are the number of points and line segments, respectively. We then build a random rotation matrix; the translation vector is taken to be the mean vector of all the points, as done in [45]. From the last $2 n_{1}$ generated points, we form $n_{1}$ 
(a) Nonplanar: $\operatorname{PnP}\left(n_{\mathrm{p}}=6\right), \operatorname{PnL}\left(n_{1}=10\right), \operatorname{PnPL}\left(n_{\mathrm{p}}=6, n_{1}=10\right)$
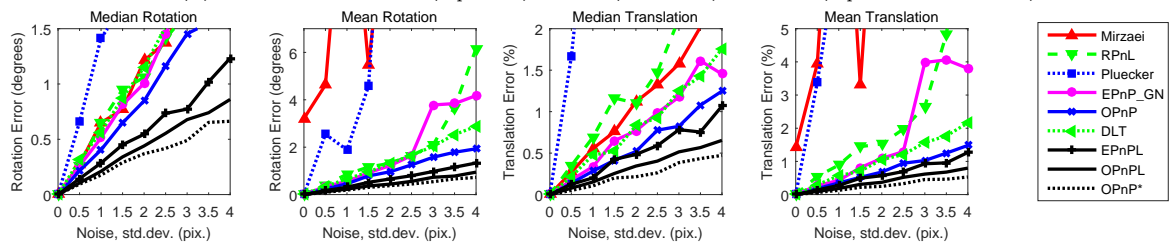

(b) Nonplanar: PnP $\left(n_{\mathrm{p}}=10\right)$, PnL $\left(n_{1}=10\right)$, PnPL $\left(n_{\mathrm{p}}=n_{1}=5\right)$
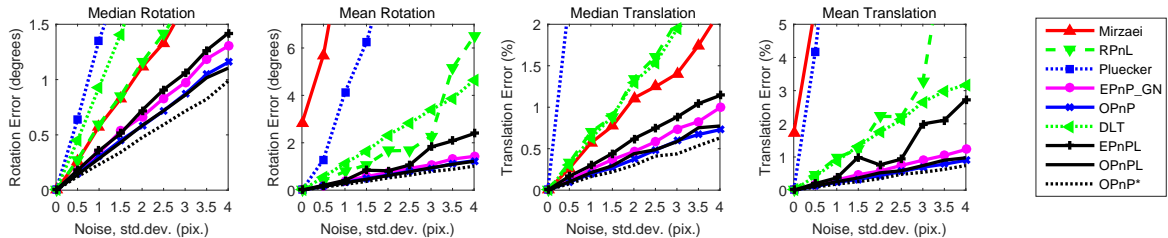

(c) Planar: PnP $\left(n_{\mathrm{p}}=6\right), \operatorname{PnL}\left(n_{1}=10\right), \operatorname{PnPL}\left(n_{\mathrm{p}}=6, n_{1}=10\right)$
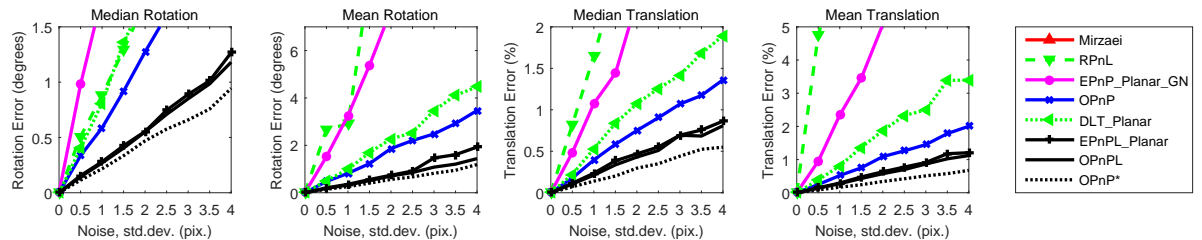

(d) Planar: PnP $\left(n_{\mathrm{p}}=10\right), \operatorname{PnL}\left(n_{1}=10\right), \operatorname{PnPL}\left(n_{\mathrm{p}}=n_{1}=5\right)$
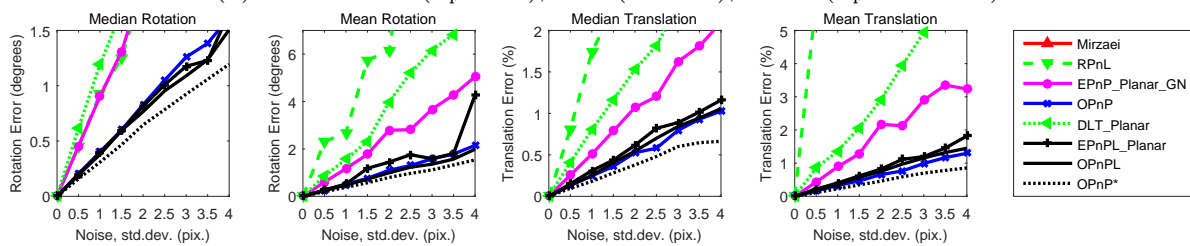

Fig. 3. Pose from point and line correspondences. Accuracy w.r.t. image noise level.

pairs, which will be the $3 \mathrm{D}$ reference segments $\left(\mathbf{P}\right.$ and $\mathbf{Q}$ in Fig. 2). The $n_{\mathrm{p}} 3 \mathrm{D}$ points and $2 n_{1}$ endpoints are then projected onto the image, and perturbed by Gaussian noise. For each 3D line we generate two more points on the same line $\left(\mathbf{P}_{\mathrm{d}}\right.$ and $\mathbf{Q}_{\mathrm{d}}$ in Fig. 2), by randomly shifting $\mathbf{P}$ and $\mathbf{Q}$, and project them onto the image and corrupt with noise $\left(\mathbf{p}_{\mathrm{d}}\right.$ and $\mathbf{q}_{\mathrm{d}}$ in Fig. 2). In the following, unless said otherwise, noise standard deviation is set to 1 pixel, the segment length is set to a uniformly random value within the interval $[1.5,4.5]$, and the segment shift is also taken randomly within $[-2,2]$.

In all quantitative results, we will provide the absolute error (in degrees) for rotation, and the relative error (\%) for translation. All plots are created by running 500 independent simulations and report the mean and median errors.

Regarding state-of-the-art, we compare against the following PnL algorithms: RPnL [44], Mirzaei [28] and Pluecker [33]. As for PnP methods we will include EPnP [24] and OPnP [45]; and the DLT proposed in [17] for the PnPL.Our 

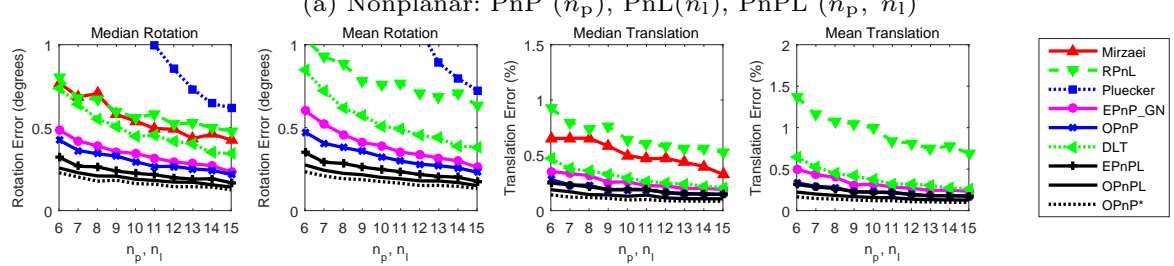

(b) Nonplanar: PnP $\left(n_{\mathrm{p}}\right), \operatorname{PnL}\left(n_{1}\right), \operatorname{PnPL}\left(n_{\mathrm{p}}^{\prime}=n_{1}^{\prime}=0.5 n_{\mathrm{p}}=0.5 n_{1}\right)$
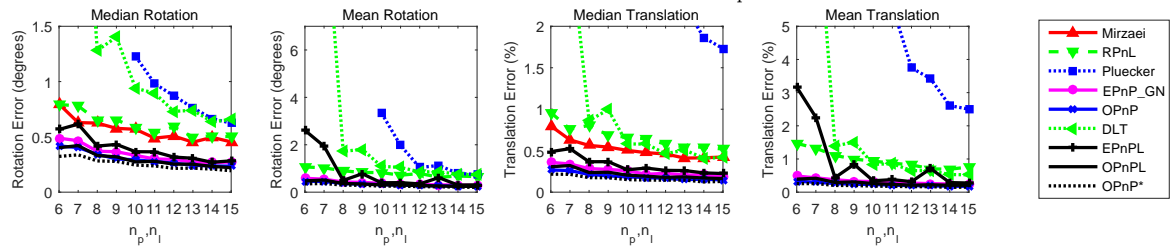

(c) Planar: PnP $\left(n_{\mathrm{p}}\right), \operatorname{PnL}\left(n_{1}\right), \operatorname{PnPL}\left(n_{\mathrm{p}}, n_{1}\right)$
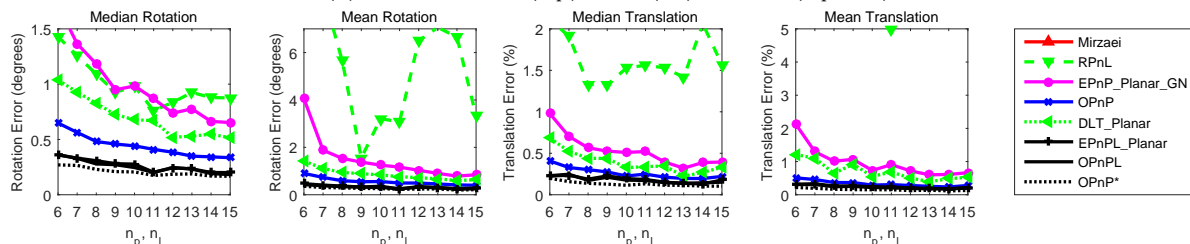

(d) Planar: PnP $\left(n_{\mathrm{p}}\right)$, PnL $\left(n_{1}\right), \operatorname{PnPL}\left(n_{\mathrm{p}}^{\prime}=n_{1}^{\prime}=0.5 n_{\mathrm{p}}=0.5 n_{1}\right)$
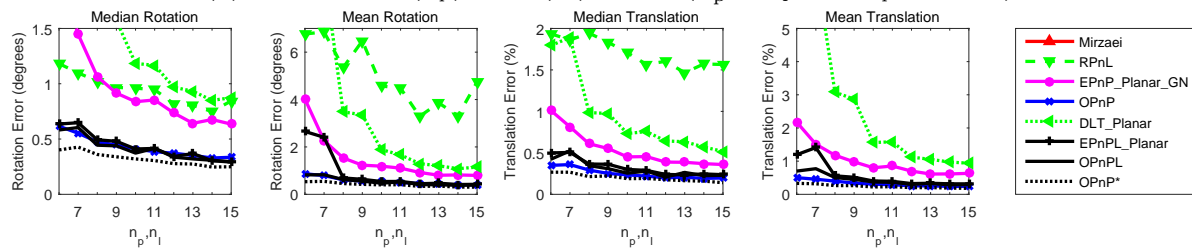

Fig. 4. Pose from point and line correspondences. Accuracy w.r.t. increasing number of point or line correspondences.

two approaches will be denoted as EPnPL and OPnPL. Additionally, we will also consider the $\mathrm{OPnP}^{*}$, which will take as input both point and line correspondences. However, for the lines we will consider the true correspondences $\left\{\mathbf{P}^{i}, \mathbf{Q}^{i}\right\} \leftrightarrow\left\{\mathbf{p}^{i}, \mathbf{q}^{i}\right\}$ instead of the correspondences $\left\{\mathbf{P}^{i}, \mathbf{Q}^{i}\right\} \leftrightarrow\left\{\mathbf{p}_{\mathrm{d}}^{i}, \mathbf{q}_{\mathrm{d}}^{i}\right\}$ (see again Fig. 2), that feed our two approaches and the rest of algorithms. Note that this is an unrealistic situation, and $\mathrm{OPnP}^{*}$ has to be interpreted as a baseline indicating the best performance one could expect.

Pose from points and lines. We consider point and line correspondences for two configurations, non-planar and planar. We evaluate the accuracy of the approaches w.r.t. the image noise in Fig. 3, and w.r.t. an increasing number of correspondences (either points, lines or points and lines) in Fig. 4. To ensure fairness between PnP, PnL and PnPL algorithms we analyze two situations: 
(a) Nonplanar: PnL $\left(n_{1}=10\right), \operatorname{PnPL}\left(n_{\mathrm{p}}=0, n_{1}=10\right)$
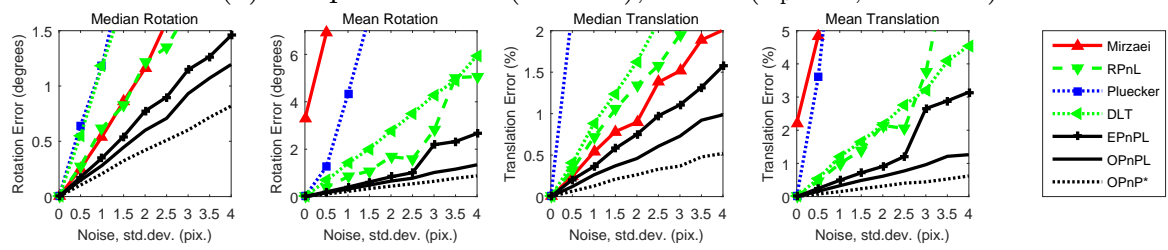

(b) Planar: PnL $\left(n_{1}=10\right)$, PnPL $\left(n_{\mathrm{p}}=0, n_{\mathrm{l}}=10\right)$
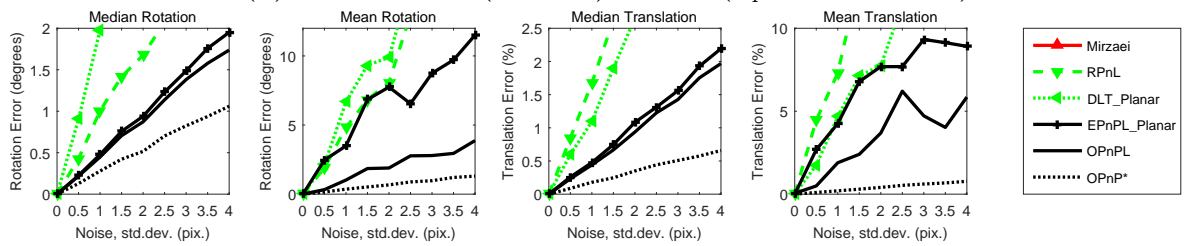

Fig. 5. Pose from only line correspondences. Accuracy w.r.t. image noise level.

(a) Different number of constraints. When evaluating accuracy w.r.t. image noise we consider $n_{\mathrm{p}}=6$ point correspondences for the $\mathrm{PnP}$ algorithms (minimum number required by $\mathrm{EPnP}), n_{1}=10$ correspondences for the $\mathrm{PnL}$ (minimum number for Pluecker [33]) and the PnPL methods use $n_{\mathrm{p}}=6$ point plus $n_{\mathrm{l}}=10$ line correspondences. The results for the non-planar and planar configurations are shown in Fig. 3-(a) and (c), respectively. As expected, the EPnPL and OP$\mathrm{nPL}$ methods are more accurate than the point only versions, because they are using additional information. Indeed, OPnPL is very close from the OPnP* baseline, indicating that line information is very well exploited. Additionally, our approaches work remarkably better than DLT (the other PnPL solution) and the rest of PnL methods. In Fig. 4-(a) and (c) we observe that the methods' accuracy w.r.t. an increasing number of points also shows that our approaches exhibit the best performances.

(b) Constant number of constraints. We also provide results of accuracy w.r.t. noise in which we limit the total number of constraints, either obtained from line or point correspondences, to a constant value $\left(n_{\mathrm{p}}=10\right.$ for PnP methods, $n_{\mathrm{l}}=10$ for PnL methods and $n_{\mathrm{p}}=5, n_{\mathrm{l}}=5$ for PnPL methods). Note that PnP algorithms will be in this case in a clear advantage, as we are feeding them with point correspondences just perturbed by noise. PnL and PnPL algorithms need to deal with weaker line correspondences which besides noisy, are less spatially constrained. Results in Fig. 3-(b) and (d) confirm this. EPnP and OPnP have largely improved their performance compared to the previous scenario, but what is very remarkable is that $\mathrm{OPnPL}$ is almost as good as its point-only version OPnP. EPnPL is more clearly behind EPnP. In any event, our solutions again perform much better than DLT and the PnL algorithms. Similar performance patterns can be observed in Fig. 4-(b) and (d) when evaluating the accuracy w.r.t. number of constraints in the non-planar case. For the planar case, our two solutions clearly outperform all other approaches, specially in rotation error. 

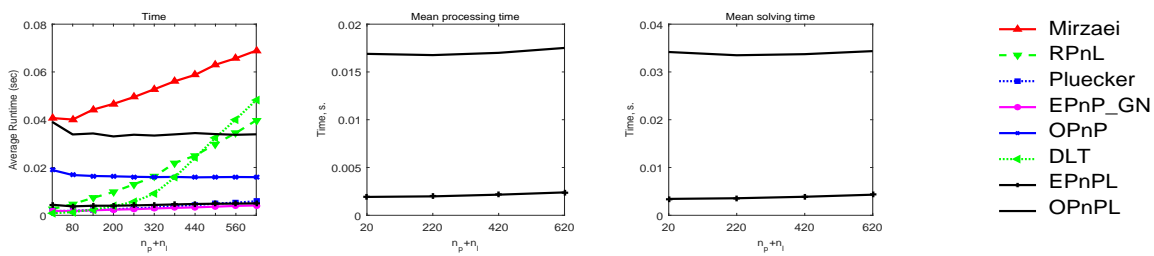

Fig. 6. Running times for increasing number of correspondences. Left: All methods. Center: Phases of our approaches. 'Processing' (center-left) includes the calculation of 2D line equations and the line correction from Sec. 3.3. 'Solving' (center-right) refers to the actual time taken to compute the pose as described in Sect. 3.4 and 3.5.

Pose from lines only. In this experiment we just compare the line-based approaches, i.e, the PnL and PnPL methods (but only fed with line correspondences). We consider $n_{1}=10$. In Fig. 5 -(a) and (b) we show the pose accuracy at increasing levels of noise, for the nonplanar and planar case. EPnPL and OPnPL perform consistently better than all other approaches, specially OPnPL. We also observed that PnL methods are very unstable under planar configurations and, in particular, Pluecker [33] did not manage to work and Mirzaei [28] yielded a very large error (out of bounds in the graphs).

Scalability. We evaluate the runtime of the methods for an increasing number of point and line correspondences. For the PnL and PnP methods we used $n_{\mathrm{p}}$ and $n_{1}$ correspondences, respectively, with $n_{\mathrm{p}}=n_{\mathrm{l}}$. The PnPL methods receive twice the number of correspondences, i.e, $n_{\mathrm{p}}+n_{\mathrm{l}}$. Fig. 6 -left shows the results, where all methods are implemented in MATLAB. As expected, the runtime of OPnPL and EPnPL is about twice the runtime of their point-only counterparts, confirming they are still $O(n)$ algorithms. This linear time is maintained even having to execute the correction scheme of the line segments described in Sect. 3.3. For this to happen, our implementations exploit efficient vectorized operations. Fig. 6right reports the time taken by our approach at different phases.

Shift of line segments. As discussed above, to simulate real situations in which a 3D reference line on the model may only be partially projected onto the input image, we enforce the detected lines to be shifted versions of the true projected lines. In this experiment, we evaluate the robustness of the PnL and PnPL algorithms to this shift, which is controlled by means of a parameter $k \in[0,1]$. $k=0$ would correspond to a non-shift, i.e., $\left\{\mathbf{p}^{i}, \mathbf{q}^{i}\right\}=\left\{\mathbf{p}_{\mathrm{d}}^{i}, \mathbf{q}_{\mathrm{d}}^{i}\right\} \cdot k=1$ would correspond to a shift of 3 units between the true projected and the detected line endpoints. Additionally, we consider another baseline, the OPnP_naive, where we feed the OPnP algorithm with the correspondences $\left\{\mathbf{P}^{i}, \mathbf{Q}^{i}\right\} \leftrightarrow\left\{\mathbf{p}_{\mathrm{d}}^{i}, \mathbf{q}_{\mathrm{d}}^{i}\right\}$. The results are shown in Fig. 7. Note that PnL methods and DLT are insensitive to the amount of shift (Mirzaei [28] occasionally fails, but this is independent on the amount of shift). This was expected, as these algorithms only consider the line directions in the image plane, and not the particular position of the lines. Our EPnPL and OPnPL approaches could be more sensitive, as we explicitly use 

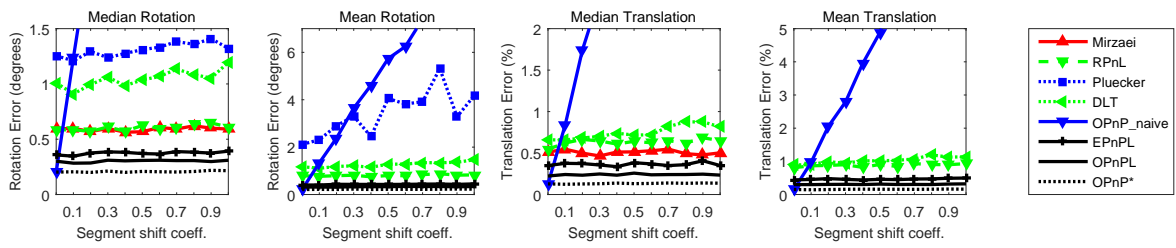

Fig. 7. Robustness to the amount of shift between the $3 \mathrm{D}$ reference lines and the projected ones. See text for details.

the position of the line in the image plane. However, the correction step we use in Sect. 3.3 gets rid of this problem. As expected OPnP_naive fails completely.

\subsection{Real Images}

We also evaluated our approach on real images of the NYU2 [39] and EPFL Castle-P19 [41] datasets. These are datasets with structured objects (indoor and man-made scenarios) and with a large amount of straight lines and repetitive patterns that will benefit of a joint point and line based approach. For these experiments we will only evaluate the point based OPnP and the OPnPL, either using only lines or lines with points.

We implemented a standard structure from motion pipeline on triplets of images for building the 3D models. Details are provided in the supplemental material. One of the images of the triplet is then taken to be the reference and another the test image. SIFT feature points are detected in both images and line features are detected and represented using the recent scale invariant line descriptor [43], shown to be adequate for wide baseline matching. The test image is matched against the model, using RANSAC with P3P for the point-only case; and using RANSAC with OPnPL for a combination of four points or lines (4 lines, 3 lines/1 point, etc.) The final pose is computed using the points within the concensus for the OPnP. For the OPnPL we consider the concensus made of both points and lines and the concensus made of only lines.

Figure 8 reports sample images of both datasets, including both quantitative and qualitative results. As can be seen, these type of scenarios (repetitive patterns in the castle, many straight and planar surfaces, low textured areas), are situations in which the point-based approaches are prone to fail, and where lines and lines+points methods perform more robustly.

\section{Conclusion}

In this paper we have proposed an approach to integrate 3D-to-2D line correspondences within the formulation of two state-of-the-art $\mathrm{PnP}$ algorithms, allowing them to indistinctly treat points, lines or a combination of them. In order to do so, we introduce an algebraic line error that is formulated in terms of the 


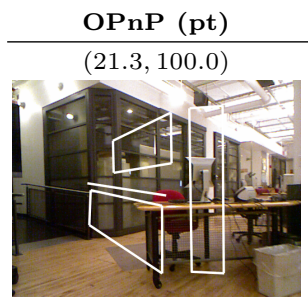

$(1.3,33.8)$

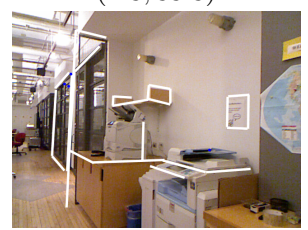

$(3.2,100.0)$

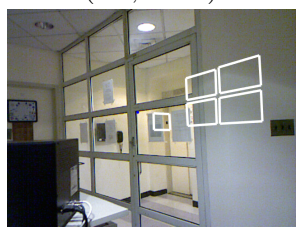

$(31.8,162.2)$

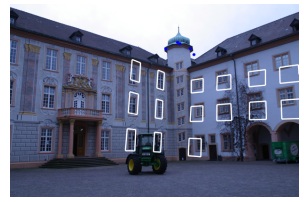

OPnPL (lin) $/(p t+l i n)$

$(9.3,30.5) /(9.5,28.2)$

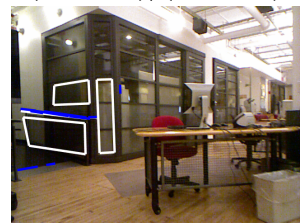

$(0.6,9.1) /(0.2,2.6)$

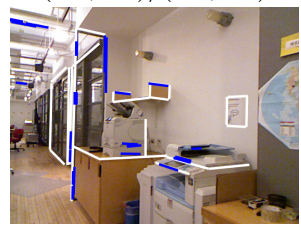

$(0.8,3.3) /(0.9,5.6)$

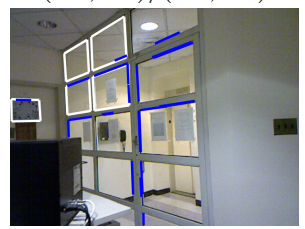

$(1.3,6.8) /(1.3,6.8)$

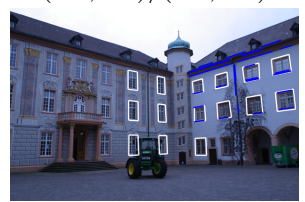

OPnP (pt)

$(61.5,605.4)$

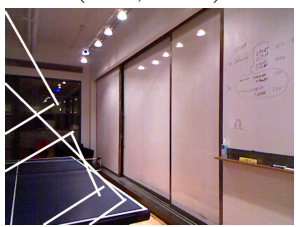

$(118.2,328.0)$

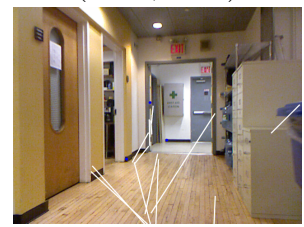

$(9.5,100.0)$

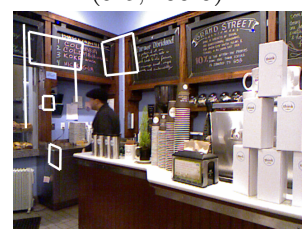

$(1.3,22.0)$

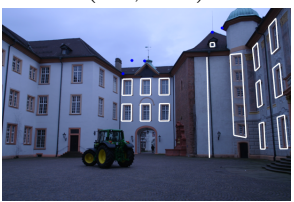

OPnPL (lin) $/(p t+$ lin $)$

$(0.4,6.0) /(0.3,5.7)$

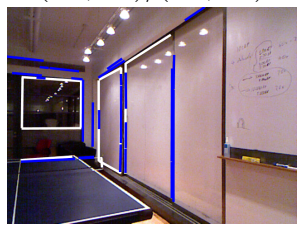

$(2.7,11.5) /(2.7,11.3)$

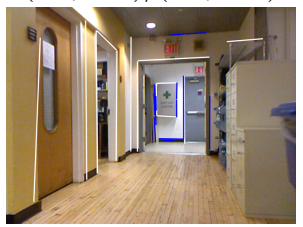

$(0.7,3.0) /(0.2,1.3)$

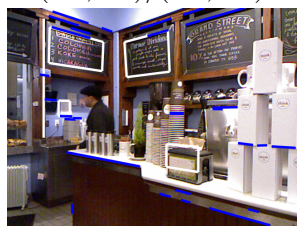

$(0.9,10.1) /(0.2,2.3)$

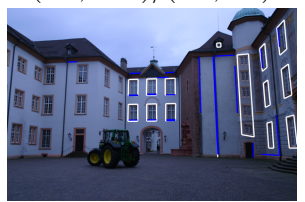

Fig. 8. Pose estimation on the NYU2 and Castle (last row) datasets, for OPnP and OPnPL. The numbers on top of each image indicate the rotation and translation error as pairs (Rot. Err. (deg), Translation Error (\%)). For the OPnPL we report both the error of the only line case, and of the case with joint points and lines. The lines of the $3 \mathrm{D}$ model are reprojected onto the images using the estimated pose. For the OPnPL we reproject the lines using the pose estimated using both points and lines.

line endpoints and which is robust to large shifts between their 3D positions and its $2 \mathrm{D}$ projection. This reproduces the situation that occurs in practice, where 3D model lines are just partially observed in the image due to occlusions or mis-detections. We extensively evaluate our algorithms on both synthetic and real images, showing a boost in performance w.r.t. other approaches, either those that only use lines, points or a combination of both. Additionally, our approach retains the $O(n)$ capabilities of the $\mathrm{PnP}$ algorithms we build upon, making them appropriate for real time computations in structure-from-motion frameworks, that since to date have mostly exploited point correspondences. We plan to explore this line in the near future. 


\section{References}

1. Abdel-Aziz, Y.: Direct linear transformation from comparator coordinates in closerange photogrammetry. In: ASP Symposium on Close-Range Photogrammetry in Illinois, 1971 (1971)

2. Ansar, A., Daniilidis, K.: Linear pose estimation from points or lines. Pattern Analysis and Machine Intelligence, IEEE Transactions on 25(5), 578-589 (2003)

3. Bay, H., Tuytelaars, T., Van Gool, L.: Surf: Speeded up robust features. In: Computer vision-ECCV 2006, pp. 404-417. Springer (2006)

4. Bujnak, M., Kukelova, Z., Pajdla, T.: A general solution to the p4p problem for camera with unknown focal length. In: Computer Vision and Pattern Recognition, 2008. CVPR 2008. IEEE Conference on. pp. 1-8. IEEE (2008)

5. Chen, H.H.: Pose determination from line-to-plane correspondences: existence condition and closed-form solutions. In: Computer Vision, 1990. Proceedings, Third International Conference on. pp. 374-378. IEEE (1990)

6. DeMenthon, D., Davis, L.S.: Exact and approximate solutions of the perspectivethree-point problem. IEEE Transactions on Pattern Analysis \& Machine Intelligence (11), 1100-1105 (1992)

7. Dhome, M., Richetin, M., Lapreste, J.T., Rives, G.: Determination of the attitude of $3 \mathrm{~d}$ objects from a single perspective view. Pattern Analysis and Machine Intelligence, IEEE Transactions on 11(12), 1265-1278 (1989)

8. Ess, A., Neubeck, A., Van Gool, L.J.: Generalised linear pose estimation. In: BMVC. pp. 1-10 (2007)

9. Ferraz, L., Binefa, X., Moreno-Noguer, F.: Very fast solution to the pnp problem with algebraic outlier rejection. In: Proceedings of the Conference on Computer Vision and Pattern Recognition (CVPR). pp. 501-508 (2014)

10. Fiore, P.D.: Efficient linear solution of exterior orientation. IEEE Transactions on Pattern Analysis \& Machine Intelligence (2), 140-148 (2001)

11. Fischler, M.A., Bolles, R.C.: Random sample consensus: a paradigm for model fitting with applications to image analysis and automated cartography. Communications of the ACM 24(6), 381-395 (1981)

12. Gao, X.S., Hou, X.R., Tang, J., Cheng, H.F.: Complete solution classification for the perspective-three-point problem. Pattern Analysis and Machine Intelligence, IEEE Transactions on 25(8), 930-943 (2003)

13. Garro, V., Crosilla, F., Fusiello, A.: Solving the pnp problem with anisotropic orthogonal procrustes analysis. In: 2012 Second International Conference on 3D Imaging, Modeling, Processing, Visualization \& Transmission. pp. 262-269. IEEE (2012)

14. Grunert, J.A.: Das pothenotische problem in erweiterter gestalt nebst u ber seine anwendungen in geoda sie. In: Grunerts Archiv fu r Mathematik und Physik (1841)

15. Haralick, R.M., Lee, C.n., Ottenburg, K., Nölle, M.: Analysis and solutions of the three point perspective pose estimation problem. In: Computer Vision and Pattern Recognition, 1991. Proceedings CVPR'91., IEEE Computer Society Conference on. pp. 592-598. IEEE (1991)

16. Harris, C., Stephens, M.: A combined corner and edge detector. In: Alvey vision conference. vol. 15, p. 50. Citeseer (1988)

17. Hartley, R., Zisserman, A.: Multiple view geometry in computer vision. Cambridge university press (2003)

18. Hesch, J.A., Roumeliotis, S.I.: A direct least-squares (dls) method for pnp. In: Computer Vision (ICCV), 2011 IEEE International Conference on. pp. 383-390. IEEE (2011) 
19. Horaud, R., Conio, B., Leboulleux, O., Lacolle, L.B.: An analytic solution for the perspective 4-point problem. In: Computer Vision and Pattern Recognition, 1989. Proceedings CVPR'89., IEEE Computer Society Conference on. pp. 500-507. IEEE (1989)

20. Kneip, L., Li, H., Seo, Y.: Upnp: An optimal o (n) solution to the absolute pose problem with universal applicability. In: Computer Vision-ECCV 2014, pp. 127 142. Springer (2014)

21. Kneip, L., Scaramuzza, D., Siegwart, R.: A novel parametrization of the perspective-three-point problem for a direct computation of absolute camera position and orientation. In: Computer Vision and Pattern Recognition (CVPR), 2011 IEEE Conference on. pp. 2969-2976. IEEE (2011)

22. Kuang, Y., Astrom, K.: Pose estimation with unknown focal length using points, directions and lines. In: Proceedings of the IEEE International Conference on Computer Vision. pp. 529-536 (2013)

23. Kuang, Y., Zheng, Y., Astrom, K.: Partial symmetry in polynomial systems and its applications in computer vision. In: Proceedings of the IEEE Conference on Computer Vision and Pattern Recognition. pp. 438-445 (2014)

24. Lepetit, V., Moreno-Noguer, F., Fua, P.: Epnp: An accurate o(n) solution to the pnp problem. International Journal of Computer Vision 81(2), 155-166 (2009)

25. Li, S., Xu, C., Xie, M.: A robust o (n) solution to the perspective-n-point problem. Pattern Analysis and Machine Intelligence, IEEE Transactions on 34(7), 1444-1450 (2012)

26. Lowe, D.G.: Distinctive image features from scale-invariant keypoints. International journal of computer vision 60(2), 91-110 (2004)

27. Lu, C.P., Hager, G.D., Mjolsness, E.: Fast and globally convergent pose estimation from video images. Pattern Analysis and Machine Intelligence, IEEE Transactions on 22(6), 610-622 (2000)

28. Mirzaei, F.M., Roumeliotis, S., et al.: Globally optimal pose estimation from line correspondences. In: Robotics and Automation (ICRA), 2011 IEEE International Conference on. pp. 5581-5588. IEEE (2011)

29. Moreno-Noguer, F.: Deformation and illumination invariant feature point descriptor. In: Computer Vision and Pattern Recognition (CVPR), 2011 IEEE Conference on. pp. 1593-1600. IEEE (2011)

30. Nakano, G.: Globally optimal dls method for pnp problem with cayley parameterization. In: British Machine Vision Conference 2015, Proceedings of. BMVA (2015)

31. Navab, N., Faugeras, O.: Monocular pose determination from lines: Critical sets and maximum number of solutions. In: Computer Vision and Pattern Recognition, 1993. Proceedings CVPR'93., 1993 IEEE Computer Society Conference on. pp. 254-260. IEEE (1993)

32. Penate-Sanchez, A., Andrade-Cetto, J., Moreno-Noguer, F.: Exhaustive linearization for robust camera pose and focal length estimation. IEEE Transactions on Pattern Analysis \& Machine Intelligence (10), 2387-2400 (2013)

33. Pribyl, B., Zemck, P., et al.: Camera pose estimation from lines using plcker coordinates. In: British Machine Vision Conference 2015, Proceedings of. BMVA (2015)

34. Quan, L., Lan, Z.: Linear n-point camera pose determination. Pattern Analysis and Machine Intelligence, IEEE Transactions on 21(8), 774-780 (1999)

35. Ramalingam, S., Bouaziz, S., Sturm, P.: Pose estimation using both points and lines for geo-localization. In: Robotics and Automation (ICRA), 2011 IEEE International Conference on. pp. 4716-4723. IEEE (2011) 
36. Rosten, E., Drummond, T.: Machine learning for high-speed corner detection. In: Computer Vision-ECCV 2006, pp. 430-443. Springer (2006)

37. Rublee, E., Rabaud, V., Konolige, K., Bradski, G.: Orb: an efficient alternative to sift or surf. In: Computer Vision (ICCV), 2011 IEEE International Conference on. pp. 2564-2571. IEEE (2011)

38. Schweighofer, G., Pinz, A.: Globally optimal o (n) solution to the pnp problem for general camera models. In: BMVC. pp. 1-10 (2008)

39. Silberman, N., Hoiem, D., Kohli, P., Fergus, R.: Indoor segmentation and support inference from rgbd images. In: Computer Vision-ECCV 2012, pp. 746-760. Springer (2012)

40. Simo-Serra, E., Trulls, E., Ferraz, L., Kokkinos, I., Fua, P., Moreno-Noguer, F.: Discriminative learning of deep convolutional feature point descriptors. In: Proceedings of the International Conference on Computer Vision (ICCV) (2015)

41. Strecha, C., von Hansen, W., Gool, L.V., Fua, P., Thoennessen, U.: On benchmarking camera calibration and multi-view stereo for high resolution imagery. In: Computer Vision and Pattern Recognition, 2008. CVPR 2008. IEEE Conference on. pp. 1-8. IEEE (2008)

42. Triggs, B.: Camera pose and calibration from 4 or 5 known 3 d points. In: Computer Vision, 1999. The Proceedings of the Seventh IEEE International Conference on. vol. 1, pp. 278-284. IEEE (1999)

43. Verhagen, B., Timofte, R., Van Gool, L.: Scale-invariant line descriptors for wide baseline matching. In: Applications of Computer Vision (WACV), 2014 IEEE Winter Conference on. pp. 493-500. IEEE (2014)

44. Zhang, L., Xu, C., Lee, K.M., Koch, R.: Robust and efficient pose estimation from line correspondences. In: Computer Vision-ACCV 2012, pp. 217-230. Springer (2013)

45. Zheng, Y., Kuang, Y., Sugimoto, S., Astrom, K., Okutomi, M.: Revisiting the pnp problem: A fast, general and optimal solution. In: Computer Vision (ICCV), 2013 IEEE International Conference on. pp. 2344-2351. IEEE (2013) 